\title{
Safety and efficacy of intravenous hydralazine and labetalol for the treatment of asymptomatic hypertension in hospitalized patients: a systematic review
}

\author{
Jocelyn Cawoski ${ }^{1}$, Katie DeBiasio ${ }^{1}$, Scott Donnachie ${ }^{1}$, Elizabeth Timanus ${ }^{1}$, David \\ Zimmerman $^{1}$, Anthony Guarascio ${ }^{1}$, Courtney Montepara ${ }^{1}$, Jordan Covvey ${ }^{1}$, and Branden \\ Nemecek $^{1}$ \\ ${ }^{1}$ Duquesne University
}

October 30, 2020

\begin{abstract}
Background: Current guidelines for the management of asymptomatic hypertension (HTN) in the inpatient setting recommend the use of oral antihypertensives. However, in clinical practice, intravenous (IV) antihypertensives are commonly utilized with little supporting evidence. The objective of this study was to evaluate literature examining the safety/efficacy of IV hydralazine and labetalol in hospitalized patients with non-emergent, asymptomatic HTN. Methods: The PRISMA guidelines were utilized to structure the systematic review. A search strategy composed of drug-, inpatient-, and HTN-related terms was conducted utilizing PubMed, Embase, and Scopus databases through May 2020. Full-text, English-language articles describing IV labetalol and/or hydralazine use for non-emergent HTN in an inpatient setting that focused on clinical outcomes (i.e. vitals, adverse effects, healthcare utilization) were included. Identified studies were screened/extracted using DistillerSR by two reviewers at each stage, and studies were evaluated qualitatively for the presence of bias. Results: From 3362 records identified in the search, a final set of 10 articles were identified. Four studies focused on labetalol (40\%), five studies on hydralazine and labetalol (50\%), and one study on hydralazine (10\%). The included studies presented a variety of outcomes, but several trends were identified, including reduction in average blood pressure in eight (80\%) studies, a risk of adverse effects in six (60\%), and increased length of stay in one (10\%). Discussion: The studies identified in this review raise concerns regarding the safety of IV hydralazine and labetalol in non-emergent HTN. Despite relatively broad clinical experience with these drugs, experimental investigations regarding their utility are recommended.
\end{abstract}

\section{Hosted file}

IJCP - main document.pdf available at https://authorea.com/users/371533/articles/489847safety-and-efficacy-of-intravenous-hydralazine-and-labetalol-for-the-treatment-ofasymptomatic-hypertension-in-hospitalized-patients-a-systematic-review 


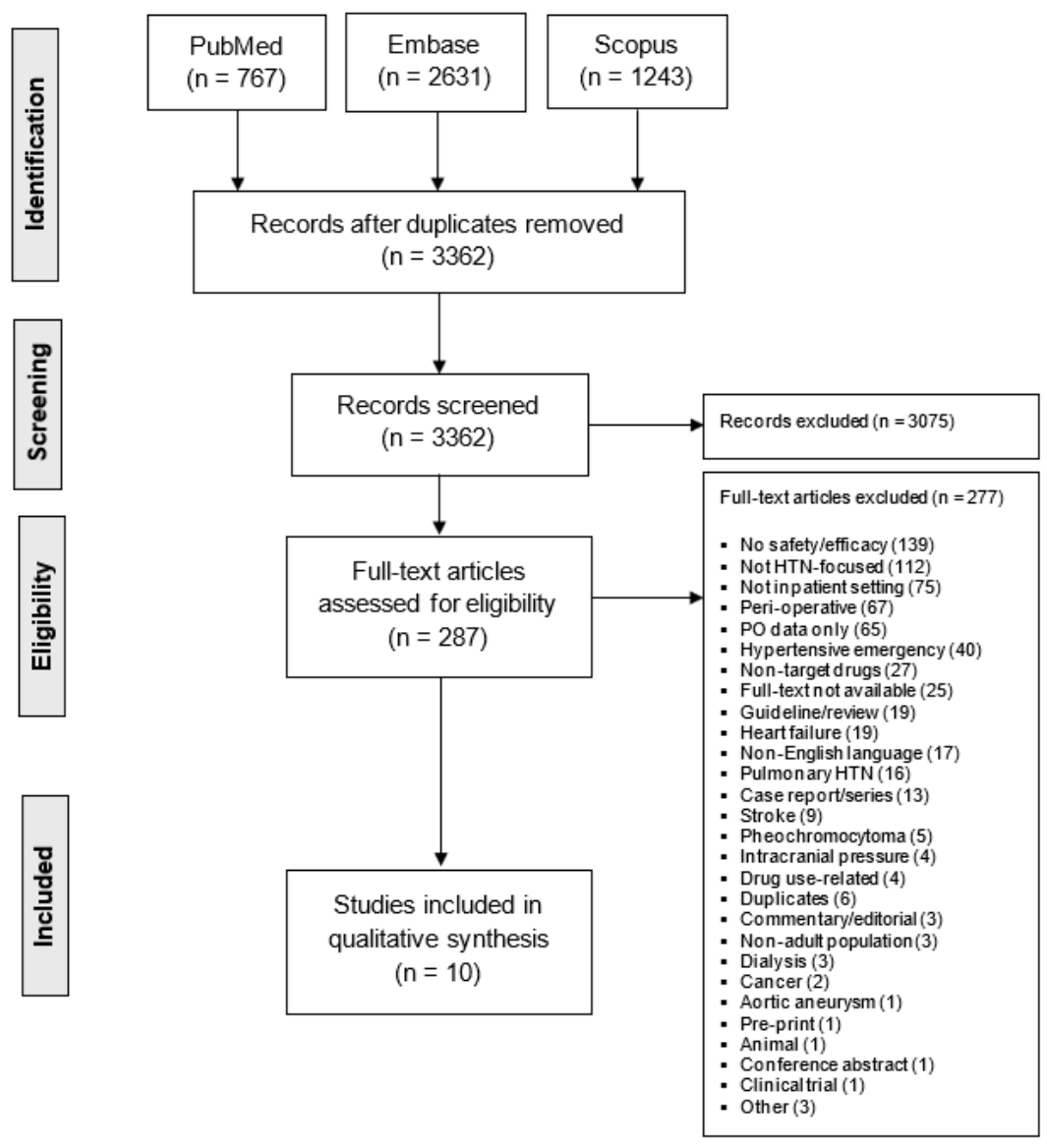

